\title{
Comunicación, ciudadanía y cambio social. Diseño de un modelo de investigación y acción para democratizar la comunicación desde la noción de reforma mediática*
}

\author{
Communication, Citizenship and Social Change. Design of a Research and Action Model to Democratize the \\ Communication based on the Notion of Media Reform \\ Comunicação, cidadania e mudança social. Desenho de um modelo de pesquisa e ação para democratizar a \\ comunicação desde a noção de reforma mediática
}

Alejandro Barranquero Carretero ${ }^{\mathrm{a}}$

Universidad Carlos III de Madrid, España

abarranq@hum.uc3m.es

ORCID: http://orcid.org/0000-0002-9264-9389
DOI: https://doi.org/10.11144/Javeriana.syp38-75.cccs

Fecha de recepción: 11 Junio 2019

Fecha de aprobación: 15 Julio 2019

Fecha de publicación: 15 Noviembre 2019

\section{Resumen:}

Este artículo intenta diseñar un modelo de análisis e investigación y acción basado en las distintas dimensiones y objetos de estudio que comprende la relación entre comunicación, ciudadanía y cambio social. Partiendo de la revisión documental de literatura de referencia, se plantea un diálogo entre la comunicación para el desarrollo y el concepto de reforma mediática, una noción que ayuda a complejizar el campo, integrando aportes de la teoría de los movimientos sociales y la economía política de la comunicación. En torno a la pregunta de "qué puede hacer la ciudadanía organizada para reformar la comunicación", el modelo integra diferentes dimensiones de la participación y distintas acciones de reformas desde dentro y fuera del sistema de medios.

Palabras clave: comunicación para el cambio social, reforma mediática, economía política de la comunicación, movimientos sociales, educomunicación.

\begin{abstract}
:
This article intends to design an analysis and research and action model based on the different dimensions and study objects involved in the relationship between communication, citizenship and social change. Starting by reviewing some documents from the referenced literature, a dialogue is proposed between communication for development and the concept of media reform, a notion that enables a more complex study of this field. It integrates contributions from the social movement theory and the political economy of communication. Regarding the question "What can organized citizens do in order to reform the communication?" this model integrates different dimensions of participation and different reform actions both inside and outside the media system. Keywords: communication for social change, media reform, political economy of communication, social movements, educommunication.
\end{abstract}

\section{Resumo:}

Este artigo visa desenhar um modelo de análise e pesquisa e ação baseado nas diversas dimensões e objetos de estudo que inclui a relação entre comunicação, cidadania e mudança social. Partindo da revisão documental de literatura de referencia, propõe-se um diálogo entre a comunicação para o desenvolvimento e conceito de reforma mediática, uma noção que ajuda a complexar o campo, integrando contribuições da teoria dos movimentos sociais e a economia política da comunicação. Em torno da pergunta de "o que a cidadania organizada pode fazer para reformar a comunicação", o modelo integra diferentes dimensões da participação e diferentes ações de reformas desde dentro e fora do sistema de mídia.

Palavras-chave: comunicação para a mudança social, reforma mediática, economia política da comunicação, movimentos sociais, educomunicação.

Notas de autor:

a Autor de correspondencia. Correo electrónico: abarranq@hum.uc3m.es 


\section{Introducción}

Históricamente, la comunicación para el desarrollo o para el cambio social (en adelante CDCS) se ha caracterizado por ser un área prolífica en conceptos, teorías y abordajes metodológicos (Gumucio y Tufte, 2006; Lie y Servaes, 2015). Sin embargo, una aproximación a la literatura evidencia que en su seno conviven planteamientos epistemológicos muy distintos e incluso opuestos. Esto la convierte en uno de los campos de estudio más fragmentados de la comunicación, en especial si se la compara con áreas más cohesionadas como los estudios de redacción periodística, la comunicación política o los trabajos sobre recepción y audiencias. Esta dispersión tiene que ver, por un lado, con la ausencia de un objeto teórico bien definido y delimitado, un hecho que dificulta el trazado de agendas y programas conjuntos de investigación (Lakatos, 1978). Por otra parte, existen diferencias sustanciales dependiendo del contexto geográfico desde el que se aborda la materia. Es por ello que, a la manera de Ángel (2013), podemos preguntarnos si existe un solo campo de la CDCS o tantos como contextos y problemas de investigación, en especial si se observan las distintas perspectivas existentes en el Norte y el Sur del planeta.

En este trabajo, se parte de la premisa de que una subdisciplina científica se caracteriza y distingue de otras por contar con un objeto de estudio específico y bien acotado, además de por métodos especializados, metas comunes y cierto grado de institucionalidad (Gee, 2010; Krishnan, 2009). En los últimos años, la CDCS ha avanzado mucho en sus procesos de institucionalización, algo que se percibe en la multiplicación de compendios y manuales (Gumucio y Tufte, 2006; Servaes, 2019; Wilkins, Tufte y Obregón, 2014) o en el progresivo reconocimiento por parte de las distintas organizaciones del desarrollo: agencias de cooperación, organizaciones no lucrativas, movimientos sociales, etc. También existe un consenso acerca de sus objetivos y metas - profundizar en los derechos humanos, la justicia social y ambiental, la calidad de vida, etc.- o sobre la importancia de desarrollar metodologías propias (Rodríguez, Obregón y Vega, 2002) e indicadores de impacto y evaluación (Lennie y Tacchi, 2013; Servaes, Polk, Shi, Reilly y Yakupitijage, 2012).

En el terreno teórico, también se aprecia que la CDCS se ha ido alejando de los antiguos modelos modernizadores -cuestionados por su carácter exógeno, economicista y mediacéntrico-para adoptar una visión más compleja, participativa y endógena de los procesos comunicacionales. Ya en la práctica, la perspectiva participativa sigue ganando popularidad entre las organizaciones del desarrollo, algunas de las cuales han dejado de mirarse en el espejo de las grandes corporaciones para imitar los repertorios de acción de los movimientos sociales (Obregón y Tufte, 2017; Tufte, 2017). Asimismo, el campo se encuentra muy vivo y permeable a la incorporación de nuevos conceptos y reflexiones tales como los de voz (Couldry, 2010), escucha y empatía (Dreher, 2010; Quarry y Ramírez, 2009), reconocimiento cultural (Fraser, 2017), capacidades (Jacobson, 2016) o buen vivir (Barranquero y Sáez-Baeza, 2015).

Pero si las finalidades y los métodos se encuentran cada vez más consensuados, no se puede decir lo propio de la existencia de un objeto bien definido. De hecho, los problemas y los límites de la CDCS han sido escasamente discutidos, por lo que las aproximaciones varían desde aquellas que asimilan el área a la comunicación alternativa de los movimientos sociales -es el caso de muchos compendios latinoamericanos y españoles (Alfaro, 2006; Chaparro, 2015; Marí Sáez, 201 1; Martínez y Sierra, 2012)- hasta las miradas más macro e institucionales predominantes en el mundo anglosajón, que conciben la comunicación como una herramienta de apoyo a los programas y políticas del desarrollo (Manyozo, 2012; Melkote y Steeves, 2001).

Este trabajo se suma a la ya larga línea de estudios teóricos publicados hasta la fecha para plantear un diálogo entre la CDCS y el concepto de reforma mediática, una noción que permite complejizar los objetos de estudio mediante una mirada extensa a la teoría de los movimientos sociales y a la economía política de la comunicación. En este sentido, uno de los objetivos del presente estudio es repolitizar la pregunta que tradicionalmente ha guiado la reflexión en el campo de la CDCS: ¿qué pueden hacer los medios para promover el cambio social? En su lugar, la noción de reforma mediática nos ayuda a deshacernos del instrumentalismo implícito en dicha pregunta -los medios para el desarrollo-y plantear una nueva ecuación 
que enfatiza en el papel de la ciudadanía como sujeto activo de cambios: ¿qué puede hacer la ciudadanía para transformar la comunicación y mejorar el sistema de medios? Poniendo el foco en las políticas y en el poder de agencia ciudadana, se plantea entonces un recorrido dividido en tres etapas. En primer lugar, se revisa la historia del campo y la dificultad de definir sus objetos y límites. Posteriormente, se explicita la estrategia metodológica y se definen y acotan las potencialidades del concepto de reforma mediática. Por último, se diseña y describe un modelo de investigación y acción basado en los distintos grados de participación ciudadana, dentro y fuera del sistema de medios.

\section{De la dificultad de definir el campo y sus objetos}

La CDCS es una de las subdisciplinas más antiguas del campo de la comunicación puesto que acumula una extensa historia más de 70 años, desde las primeras propuestas teóricas y metodológicas del paradigma modernizador estadounidense (Rogers, 1962 a las críticas de la perspectiva participativa latinoamericana (Freire, 1970/2002. Dichas orientaciones se perciben casi desde los orígenes del campo, que es compartido entonces por dos regiones distintas: (1 por un lado, EE. UU. y su abordaje de tipo más institucionalista, universalista y colonial, que propone que las organizaciones de desarrollo del Norte pueden diseñar métodos e indicadores aplicables por igual a todo el Sur del planeta y (2, por otro, Latinoamérica y otras regiones del Sur del planeta, que inciden en la importancia del desarrollo integral, la participación comunitaria y las formas alternativas de comunicación popular. Esta última orientación fue la base del nuevo modelo de la comunicación para el cambio social propuesto a finales de siglo XX y que propone un modelo dialógico para que las comunidades decidan autónomamente acerca de su propio devenir (Gumucio y Tufte, 2006.

Desde ambas perspectivas, la CDCS ha ido adquiriendo dos objetos de investigación dominantes, un hecho que se percibe tanto en los textos de referencia de la materia (Gumucio y Tufte, 2006; Wilkins et al., 2014 como en las temáticas habituales de distintos monográficos publicados en tiempos recientes ${ }^{1}$ : por un lado, los procesos de comunicación estratégica de organizaciones del desarrollo como agencias de cooperación, administraciones, fundaciones, donantes u ONG de gran tamaño - un objeto calificado como comunicación de apoyo al desarrollo- y, por otro, el extenso territorio de la comunicación ciudadana y comunitaria o la comunicación alternativa para el desarrollo democrático (Beltrán, 1993. Junto a estos objetos, se sitúa también un conjunto de problemáticas que han ocupado un lugar mucho más periférico pese a estar directamente relacionadas con la CDCS. Se hace referencia a, por un lado, objetos que, con el paso del tiempo, llegaron a conformar campos de investigación propios (p. ej., educación/comunicación, alfabetización mediática o educomunicación y, por otro, a problemáticas que han sido objeto de un interés más reciente y que tienden a conformar campos autónomos aún en proceso de institucionalización (p. ej., comunicación/TIC y movimientos sociales. Asimismo, hay líneas de investigación que raramente se han adscrito o han adoptado marcos teóricos y metodológicos de la CDCS, si bien la pregunta por la relación entre comunicación, ciudadanía y cambio social es evidente en todas ellas: representaciones mediáticas de la desigualdad, vigilancia ciudadana del sistema de medios, tácticas de reapropiación y subversión de los mensajes mediáticos, etc.

Esta distribución entre objetos dominantes y periféricos se percibe también en las principales asociaciones de investigación de la comunicación a nivel mundial (IAMCR, ICA, ECREA, ALAIC, etc, en las que existen secciones de trabajo muy activas y especializadas en CDCS, ya sea desde una perspectiva más institucionalista o basal. El caso que mejor lo ejemplifica es el de la International Association for Media and Communication Research (IAMCR, que distribuye los estudios entre una sección originaria de Investigación en Comunicación Participativa (Participatory Communication Research, cada vez más centrada en la comunicación de las grandes organizaciones, y otra dedicada a Comunicación Comunitaria y Medios Alternativos (Community Communication and Alternative Media. 
Todas estas evidencias apuntan a un particular ordenamiento del conocimiento en el que intervienen, al menos, dos factores. En primer lugar, las dinámicas epistemológicas propias de la especialización científica y las sociológicas del campo académico (Bourdieu, 2003). Estas han derivado con el tiempo en la constitución de un campo cada vez más especializado en que se perciben normas, jergas y distintas posiciones de poder. En segundo lugar, la CDCS es una subdisciplina singular en comparación con otras materias, dado que se caracteriza por su carácter aplicado, su orientación ética-política y su contexto situado. En otras palabras, el campo no se limita a describir la realidad, sino que se orienta a intervenir en ella con objeto de introducir mejoras, por lo que, sus teorías suelen generarse a la zaga de las propias experiencias sobre el terreno (Beltrán, 1993). Por otra parte, sus teorías son siempre locales y situadas, es decir, los distintos contextos geográficos y temporales han acabado por conformar tradiciones epistemológicas distintas e incluso enfrentadas. Buena muestra de ello es que existen más de 50 denominaciones distintas (Ferron, 2012) para definir fenómenos parecidos: comunicación para el desarrollo o para el cambio social, comunicación comunitaria, alternativa, popular, del tercer sector, horizontal, participativa, etc. En ocasiones, dichas etiquetas solo aportan matices a la comprensión del fernómeno. Sin embargo, su proliferación expresa que nos hallamos frente a un campo prolífico y en constante proceso de renovación.

En los casos más extremos, la dispersión conceptual ha conducido a polémicas y, sobre todo, a la dificultad de establecer alianzas entre proyectos que, más allá de las diferencias, comparten una visión crítica y democratizadora de la comunicación. Los casos que ejemplifican estos desencuentros son frecuentes: el insuficiente diálogo existente entre los medios públicos y los comunitarios; las escasas alianzas que se perciben entre medios más tradicionales (como radios comunitarias) y nuevos (nativos digitales, comunidades de software libre, colectivos de ciberactivistas, etc.), o las brechas que se dan entre medios indígenas, rurales y comunitarios en algunas regiones de América Latina. Asimismo, es frecuente encontrar fracturas en relación con los modelos de organización de los distintos proyectos -más verticales o asamblearios-, las vías de financiación - más o menos abiertos a la publicidad, por ejemplo-o sus propios ámbitos de incidencia: medios feministas, ecologistas, juveniles, etc. o proyectos de orientación más reivindicativa, cultural o inclusiva (García García, 2017).

Derivado de estos factores, el campo de la CDCS se ha convertido en una especie de paraguas conceptual en exceso impreciso y en el que se enmarcan una extensa variedad de líneas de investigación: desde el universo de las radios libres y comunitarias a los programas más institucionales de transferencia tecnológica, o desde las investigaciones sobre acceso a las TIC de grupos vulnerables a estudios sobre la resistencia digital de los movimientos sociales. Por último, y aunque la etiqueta de comunicación para el cambio social siga ganando adhesiones, la comprensión del término se ha despolitizado en algunas instancias, por lo que sus usos varían en función de las comprensiones: más verticalistas u horizontales, más institucionalistas o basales, etc. En los casos más extremos, se puede incluso afirmar que la CDCS corre el riesgo de transformarse en un significante vacio (Barassi, 2016) que exprese abordajes muy distintos, e incluso opuestos, de lo que significa la relación entre comunicación, ciudadanía y transformación.

\section{Metodología y operación epistémica}

Este artículo parte de un proceso de revisión documental de bibliografía de referencia en el campo de la CDCS y en el concepto emergente de "reforma mediática", con objeto de explorar los supuestos y debates dominantes que subyacen en ambos. En la línea de Montuori (2005), se parte de la premisa de que la revisión documental no debe concebirse desde actitudes reproductivas de quien dijo qué, sino que ha de orientarse más bien a realizar una interpretación creativa de la literatura, a partir de diálogos entre el revisor y el campo y de cara a generar nuevas direcciones para la investigación y la acción. En este sentido, la finalidad última de nuestra revisión es construir un modelo de análisis que dé cuenta de la diversidad de objetos de investigación 
que comprende o que podría comprender el campo si se proyecta sobre él una mirada amplia y en clave de economía política y desafíos ciudadanos.

Se parte de la certeza de que no todas las investigaciones que se preguntan por la relación entre comunicación y transformación social se adscriben o enmarcan en la CDCS, tal y como han demostrado metainvestigaciones recientes sobre artículos académicos estadounidenses (Ogan et al., 2009 o latinoamericanos (Barranquero y Ángel, 2015). En esta línea, McAnany sugiere que las revistas de comunicación no son ni siquiera la mejor fuente para conocer lo que ocurre en el área, puesto que hay muchísimos trabajos publicados en revistas de sanidad, educación o estudios rurales de acuerdo al carácter aplicado del campo (McAnany, 2010, pp. 12-13). Por su parte, Lie y Servaes (2015) han cartografiado las subdisciplinas que componen el campo, distinguiendo entre áreas temáticas -comunicación para la salud, agrícola y rural y ambiental- y no temáticas -comunicación estratégica, participativa, de crisis y de riesgo-. Su estudio invita a repensar y a reordenar la disciplina, ampliando la mirada desde la "homogeneidad" hacia las "diferencias" y desde la "modernización" hacia asuntos relacionados con la "complejidad, la hibridación de las culturas, la (post) modernidad, el multiculturalismo, la sostenibilidad y la transdisciplinariedad” (Lie y Servaes, 2015, p. 252).

A nuestro entender, la solución para ordenar tan vasto universo de objetos y problemáticas pasa por reconducir los términos habituales de la pregunta que ha guiado el devenir del área ("¿qué pueden hacer los medios para transformar la sociedad?"), y enriquecerla con una pregunta menos centrada en los instrumentos y más en las apropiaciones y las políticas ciudadanas o, en otras palabras, “¿qué puede hacer la ciudadanía organizada para transformar las estructuras mediáticas o para la cambiar la sociedad a partir de los medios?”. Consideramos incluso que algunas conceptualizaciones recientes sobre la materia adolecen de carencias y limitaciones. Tal es el caso del popularizado concepto de comunicación para el cambio social, que se define como un proceso de diálogo público y privado a través del cual las personas definen quiénes son y qué quieren y cómo pueden obtenerlo (Gumucio y Tufte, 2006). Esta definición es incompleta por distintos supuestos (Barranquero y Sáez-Baeza, 2015), pero también porque se centra en exclusiva en los procesos participativos de comunicación ciudadana. En este sentido, la noción no comprende otras problemáticas que han estado históricamente relacionadas con el sector como: cuestiones de acceso a medios y TIC, representaciones de la ciudadanía en los medios convencionales, prácticas de educación mediática o estrategias de subversión de los sentidos hegemónicos que transmiten los medios.

Por último, la pregunta que se formula como punto de partida para ordenar los objetos está relacionada con otras dos nociones: en primer lugar, los niveles de intervención o participación ciudadana en el sistema de medios (Carpentier, 2011) y, en segundo, la reforma mediática, que valora cuáles son las vías de que dispone la ciudadanía para democratizar el sistema mediático, creando redes y alianzas (Hackett y Carroll, 2006).

\section{El concepto de reforma mediática}

El concepto de reforma mediática (media reform) se comienza a popularizar a finales de siglo XX en el ámbito anglosajón (Calabrese, 2004; McChesney, 1999; Napoli, 2007), si bien ha sido poco explorado en el entorno hispanohablante, a excepción de algunos trabajos pioneros en España (Álvarez, 2017; Tucho, 2006) o de estudios latinoamericanos que exploran el papel de la sociedad civil en la promoción del derecho a la comunicación en distintos países (Segura, 2011; Segura y Waisbord, 2016). Por otra parte, la noción apenas ha penetrado en el campo de la CDCS, puesto que aparece más vinculada al ámbito de la economía política de la comunicación (McChesney, 1999) y, en menor medida, al estudio de los movimientos sociales (Hackett y Carroll, 2006).

No obstante, se considera que el concepto tiene un enorme potencial para enriquecer y repolitizar la CDCS al abrir un diálogo con dichos marcos teóricos, necesidad que ha sido detectada en trabajos recientes 
como el de Rodríguez, Ferron y Shamas (2014), que invitan a complejizar la comunicación alternativa mediante una mirada amplia a los contextos históricos y a la economía política, en especial a fin de evitar el tecnodeterminismo que caracteriza a muchos estudios recientes de comunicación y movimientos sociales. Por su parte, Florencia Enghel (2015) considera que la economía política ayuda a valorar las prácticas transformadoras desde los condicionantes que impone el capitalismo globalizado, mientras que Tufte (2017) y Obregón y Tufte (2017) apuestan por reforzar el diálogo con las teorías de la acción colectiva y visiones más basales y comunitarias. Desde estas recomendaciones, se considera que la noción de reforma mediática tiene cuatro potencialidades analíticas.

En primer lugar, subraya la importancia de construir grandes alianzas en favor de la democratización de las comunicaciones, lo que incluye una valoración de las distintas organizaciones y actores que manejan una visión crítica del sistema de medios: desde instancias más institucionales y profesionalizadas (políticos, reguladores, académicos, medios convencionales, etc.) a entidades de la sociedad civil como ONG y movimientos sociales. El reclamo de una democracia mediática (Pickard, 2015) incluye, a su vez, diferentes demandas: desde el refuerzo de la libertad de expresión y el derecho a la comunicación ${ }^{2}$ a la reivindicación de políticas de apoyo a medios públicos y comunitarios y leyes antimonopolio para asegurar la diversidad cultural en los medios privados. En algunos momentos, estas reclamaciones han llegado a conformar movimientos sociales centrados en exclusiva en la comunicación, que se caracterizan por sus contornos globales (Padovani y Calabrese, 2014; Stephansen, 2017) y por imbricarse con un conjunto más amplio de luchas en sectores como la salud, la educación, los derechos civiles o la protección ambiental (McChesney, 2016).

En segundo lugar, la literatura se pregunta por las interconexiones entre medios, Estado y poder económico, lo que ayuda a enmarcar la CDCS en el contexto más amplio de la economía política de la comunicación. Se parte de la premisa de que el sistema dominante de medios se organiza y refuerza a partir de redes de monopolios interconectados que son, a su vez, interdependientes de Estados que regulan y, sobre todo, desregulan los medios. Los Estados legitiman sus acciones desde la excusa de que el adelgazamiento de lo público y el libre flujo de información a manos de empresas privadas favorece una oferta amplia y diversa de contenidos (Zallo, 2016). La única manera de desafiar los excesos de un capitalismo mediático global y organizado en redes es, precisamente, armar redes de ciudadanía organizada (McChesney, 2016). En este sentido, el concepto se conecta con la teoría de los movimientos sociales por cuanto explora cuáles son las principales ventanas de oportunidad para obtener reformas mediáticas: desde crisis económicas y crisis orgánicas del sistema en un sentido "gramsciano" (Calabrese, 2004) a intereses contradictorios y el lucha de las élites (Freedman, 2014).

En tercer lugar, es habitual encontrar una teorización acerca de los espacios desde los cuales ejercer la reforma, muchos de los cuales rebasan los medios tradicionales para explorar el activismo digital en sus diferentes versiones. En esta línea, Hackett (2000) plantea que la reforma puede ejercerse dentro o fuera de los medios, es decir, mediante reformas internas (como normas y organismos de autorregulación, formación crítica de los profesionales, etc.), o a partir de mecanismos externos de fiscalización y rendición de cuentas: consejos audiovisuales, observatorios, asociaciones de usuarios, etc. (Hackett, 2000). Otros autores delimitan los espacios en relación con el eje público, privado o comunitario (Crowther, Thibault, Salamon y King, 2016), o con respecto a las relaciones que se dan dentro y fuera del Estado. En esta línea, Fenton y Freedman (2014) y Freedman y Obar (2016) señalan que existen, al menos, tres tipos de reforma mediática: las correspondientes a la "crítica", o el hecho de "conocer los medios" (su ideología, sus condicionantes políticos y económicos, etc.); la "práctica" o la dimensión de "ser los medios" y fomentar experiencias comunicacionales autónomas para cubrir las parcelas descuidadas por los mainstream y, por último, las "políticas” o el eje de "transformar los medios", que implica establecer relaciones y lobbies con respecto al Estado a fin de garantizar políticas y regulaciones favorables.

Por último, la literatura ha descrito casos de éxito de estas coaliciones a la hora de favorecer políticas públicas o activar un horizonte común de luchas (Freedman, 2014; Freedman, Obar, Martens y McChesney, 
2016; Padovani y Calabrese, 2014; Pickard y Yang, 2017). En este sentido, algunos estudios se remontan a la descripción de casos históricos paradigmáticos como el de la Comisión Hutchins o movimientos en los propios orígenes de la regulación radiofónica en EE. UU. (Fones-Wolf, 2006), mientras que otros destacan hitos recientes como la aprobación de normas para garantizar la neutralidad de la red (Pickard, 2015) o luchas relacionadas con la propiedad intelectual: Stop Online Piracy Act (SOPA) en EE. UU., Ley Sinde en España, etc. (Sarikakis y Rodríguez-Amat, 2014). En Latinoamérica, algunos teóricos han destacado el trabajo reciente de la Coalición por una Comunicación Democrática, que redactó 21 puntos para una nueva Ley de Servicios de Comunicación Audiovisual (2009) en Argentina (Segura y Waisbord, 2016). La tabla 1 resume algunos hitos históricos y contemporáneos de la reforma mediática.

TABLA 1

Hitos históricos y contemporáneos de reforma mediática

\begin{tabular}{|c|c|c|c|c|}
\hline Nombre de la coalición y web & Principal hito & Lugar y fecha & Impulsores & Descripción \\
\hline $\begin{array}{l}\text { Commission on Freedom of the } \\
\text { Press (Hutchins Commission) }\end{array}$ & $\begin{array}{l}\text { Informe Hutchins } \\
(1947)\end{array}$ & $\begin{array}{l}\text { EE. UU., } \\
1944-1947\end{array}$ & $\begin{array}{l}\text { Intelectuales y expertos } \\
\text { universitarios }\end{array}$ & $\begin{array}{l}\text { Contribuyó a conformar la teoria de la } \\
\text { responsabilidad social de la prensa, que cuestiona } \\
\text { el paradigma "objetivista” de la información, } \\
\text { critica la sujeción de la prensa a la publicidad y } \\
\text { las grandes empresas y propugna que el Estado } \\
\text { debe garantizar unos medios libres y conectados } \\
\text { con los intereses de la ciudadania (Sieberg, } \\
\text { Peterson y Schramm, 1956). }\end{array}$ \\
\hline $\begin{array}{l}\text { International Commission for } \\
\text { the Study of Communication } \\
\text { Problems }\end{array}$ & Informe McBride (1980) & $\begin{array}{l}\text { Mundial, } \\
1977-1980\end{array}$ & $\begin{array}{l}\text { Periodistas, escritores y } \\
\text { académicos }\end{array}$ & $\begin{array}{l}\text { Hito del Nuevo Orden Mundial de la } \\
\text { Información y la Comunicación (NOMIC), } \\
\text { orientado a reequilibrar los flujos mundiales de } \\
\text { información, el control de los monopolios y la } \\
\text { promoción de medios públicos, privados y } \\
\text { comunitarios plurales y de calidad. }\end{array}$ \\
\hline $\begin{array}{l}\text { Campaña por los Derechos de } \\
\text { la Comunicación en la Sociedad } \\
\text { de la Información (CRIS) } \\
\text { http://lac.derechos.apc.org/wsi } \\
\text { s/cris.shtml }\end{array}$ & $\begin{array}{l}\text { Contracumbres } \\
\text { ciudadanas durante la } \\
\text { realización de la Cumbre } \\
\text { Mundial de la Sociedad } \\
\text { de la Información } \\
\text { (WSIS) (Ginebra, 2003; } \\
\text { Tunisia, 2005) }\end{array}$ & $\begin{array}{l}\text { Mundial, } \\
\text { 2001-2005 }\end{array}$ & $\begin{array}{l}\text { Plataforma por los } \\
\text { Derechos de la } \\
\text { Comunicación, que } \\
\text { agrupó a distintas ONG y } \\
\text { asociaciones } \\
\text { comunicacionales }\end{array}$ & $\begin{array}{l}\text { Profundizó en la idea del derecho a la } \\
\text { comunicación, que expande la noción de } \\
\text { libertad de información al incorporar nuevos } \\
\text { supuestos como la diversidad cultural, la } \\
\text { participación ciudadana y el derecho colectivo } \\
\text { para desarrollar infraestructuras } \\
\text { comunicacionales o la protección del } \\
\text { patrimonio cultural, etc. (Hamelink, 2014). }\end{array}$ \\
\hline
\end{tabular}

Fuente: elaboración propia

* Aunque nace como Coalición para una Radiodifusión Democrática en 2004, impulsada por el Foro Argentino de Radios Comunitarias (FARCO), en 2012, decide ampliar su radio de acción y cambiar su nombre a Coalición por una Comunicación Democrática. Tras la desactivación de la ley por el nuevo presidente Mauricio Macri en 2015, la comisión ha seguido generando encuentros y aprobado documentos como los "Nuevos 21 puntos por el Derecho a la Comunicación" (3 de marzo de 2016). 
TABLA 1 (CONT.)

Hitos históricos y contemporáneos de reforma mediática

\begin{tabular}{|c|c|c|c|c|}
\hline $\begin{array}{l}\text { Nombre de la coalición y } \\
\text { web }\end{array}$ & Principal hito & $\begin{array}{l}\text { Lugary } \\
\text { fecha }\end{array}$ & Impulsores & Descripción \\
\hline $\begin{array}{l}\text { Coalición por una } \\
\text { Comunicación } \\
\text { Democrática* } \\
\text { http://www.coalicion.org.ar }\end{array}$ & $\begin{array}{l}21 \text { puntos básicos } \\
\text { para una nueva } \\
\text { Ley de } \\
\text { Radiodifusión }\end{array}$ & $\begin{array}{l}\text { Argentina, } \\
2004 \text {-... }\end{array}$ & $\begin{array}{l}\text { Sindicatos, universidades, medios } \\
\text { comunitarios, organizaciones de } \\
\text { derechos humanos, cooperativas, } \\
\text { medios independientes, etc. }\end{array}$ & $\begin{array}{l}\text { Los } 21 \text { Puntos tenian como objetivo incidir en el } \\
\text { proyecto de redacción de la Ley de Servicios de } \\
\text { Comunicación Audiovisual en Argentina (2009), } \\
\text { que sustituyó el marco regulatorio de la dictadura y } \\
\text { fue motor de discusiones regionales sobre el control } \\
\text { de los monopolios y la división proporcional del } \\
\text { espectro radioeléctrico en tres sectores: público, } \\
\text { comercial y comunitario (Segura, 2011; Segura y } \\
\text { Waisbord, 2016). }\end{array}$ \\
\hline $\begin{array}{l}\text { Internet Rights and } \\
\text { Principles Dynamic } \\
\text { Coalition, IRPC Coalition } \\
\text { http://internetrightsandpri } \\
\text { nciples.org }\end{array}$ & $\begin{array}{l}\text { Carta IRP de } \\
\text { Derechos } \\
\text { Humanos y } \\
\text { Principios de } \\
\text { Internet }\end{array}$ & $\begin{array}{l}\text { Mundial, } \\
2006 \text {... }\end{array}$ & $\begin{array}{l}\text { Plataformas civiles de justicia social } \\
\text { en medios e Internet, con } \\
\text { participación de algunos actores } \\
\text { del sector privado y representantes } \\
\text { de Gobiernos. }\end{array}$ & $\begin{array}{l}\text { Red abierta a actores públicos y comunitarios } \\
\text { vinculada al Internet Governance Forum (IGF) de } \\
\text { las Naciones Unidas. Intenta reforzar la vinculación } \\
\text { de Internet con los derechos humanos y ha } \\
\text { participado en el WSIS y en distintas cumbres de } \\
\text { Internet y gobernanza global (Franklin, 2016). }\end{array}$ \\
\hline $\begin{array}{l}\text { Foro Andaluz de Educación, } \\
\text { Comunicación y } \\
\text { Ciudadania } \\
\text { http://www.comunicaciony } \\
\text { ciudadania.org }\end{array}$ & $\begin{array}{l}\text { Propuesta } \\
\text { Legislativa sobre } \\
\text { la Futura Ley de } \\
\text { Comunicación } \\
\text { Audiovisual } \\
\text { Andaluza }\end{array}$ & $\begin{array}{l}\text { Andalucia, } \\
\text { España, } \\
2010-. .\end{array}$ & $\begin{array}{l}\text { Impulsado por la ONG CIC Batá, } \\
\text { integra académicos/as, medios } \\
\text { comunitarios, ONG de } \\
\text { comunicación y derechos } \\
\text { humanos, etc. }\end{array}$ & $\begin{array}{l}\text { Inspirado en el Informe McBride y la Ley Argentina, } \\
\text { el Foro ha desarrollado recomendaciones de cara a la } \\
\text { Ley de la Comunicación Audiovisual en Andalucía: } \\
\text { derecho a la comunicación, reconocimiento de } \\
\text { medios comunitarios, fortalecimiento de los medios } \\
\text { públicos, derecho de acceso, alfabetización } \\
\text { mediática, etc. (Prieto, 2013). }\end{array}$ \\
\hline
\end{tabular}

\section{Fuente: elaboración propia}

* Aunque nace como Coalición para una Radiodifusión Democrática en 2004, impulsada por el Foro Argentino de Radios Comunitarias (FARCO), en 2012, decide ampliar su radio de acción y cambiar su nombre a Coalición por una Comunicación Democrática. Tras la desactivación de la ley por el nuevo presidente Mauricio Macri en 2015, la comisión ha seguido generando encuentros y aprobado documentos como los “Nuevos 21 puntos por el Derecho a la Comunicación” (3 de marzo de 2016).

La lista de coaliciones de reforma mediática es amplia y no se agota en los ejemplos descritos. También hay trabajos que examinan reformas en contextos como África o Asia (El-Issawi, 2016) o que analizan desde el concepto las Contracumbres del Foro Social Mundial; las redes del periodismo alternativo (p. ej., Indymedia) y de filtraciones (p. ej., Wikileaks) o el propio ciberactivismo global de Anonymous. Por último, se puede encontrar una abundante literatura en torno a campañas y buenas prácticas de la sociedad civil como las impulsadas por la Media Reform Coalition (s. f.) en Reino Unido, o los organismos canadienses Open Media (s. f.) y The Media Democracy Project (s. f.) en Canadá (Hackett, 2014).

\section{La construcción de un modelo de análisis}

A partir del acercamiento al concepto de reforma mediática, se considera que la CDCS puede redefinir y ampliar sus objetos al preguntarse por dos dimensiones contenidas en los debates expuestos: (1) ¿qué posición adopta la ciudadanía con respecto al origen de la reforma mediática: desde el exterior o desde el interior de los medios? y (2) ¿cuál es su grado de participación en el proceso? (a) bajo: cuando la ciudadanía accede a los medios o es representada en la toma de decisiones de los mismos por parte de políticos, reguladores o periodistas, (b) medio: cuando es encarnada en organizaciones de la sociedad civil: ONG, movimientos sociales, etc. y (c) alto: cuando se apropia directamente de medios y tecnologías a fin de construir expresiones al margen del sistema público o comercial dominante. Las siguientes líneas describen con mayor profundidad los diferentes objetos de este modelo, avanzando desde reformas en el interior o en el exterior de los medios y desde un nivel bajo a uno alto de participación ciudadana. 


\section{Acceso a medios y tecnologías}

Entroncado con la tradición de la hipótesis del diferencial de conocimiento (knowledge gap) ${ }^{3}$, muy importante en los primeros estudios de CDCS, este objeto hace referencia a las investigaciones que inciden en el acceso de la población (en particular de sus grupos más vulnerables: infancia, mayores, migrantes, poblaciones rurales, etc.) a una oferta variada y plural de medios y, más recientemente, a tecnologías como Internet o telefonía móvil. En este punto, se incluyen, por ejemplo, estudios sobre brechas y desigualdades digitales, la planificación de políticas y estrategias de inclusión (p. ej., telecentros), el derecho de acceso a medios públicos, e incluso privados, desde ideales como la diversidad cultural, religiosa o lingüística.

\section{Representación en los medios}

Aunque esta parcela ha estado tradicionalmente ligada a distintas formas de análisis de contenido y discurso o a la evaluación de agendas (issues) y enfoques (frames), desde este modelo se concibe un objeto para evaluar la representación de la ciudadanía en los medios y a denunciar los estereotipos de género, clase, étnicos, geográficos, etarios, estéticos, profesionales, etc., prevalecientes en los medios. El área comprende, al menos, tres subtemas específicos: la representación de grupos vulnerables (mujeres, migrantes, etnias, minorías políticas y religiosas, infancia, mayores, discapacidad/disfuncioalidad, salud mental, etc.); el tratamiento informativo de los movimientos sociales y la sociedad organizada y la denominada comunicación del desarrollo, asociada a la cobertura de temáticas y debates del campo de la cooperación: Objetivos de Desarrollo del Milenio (ODN), Objetivos para el Desarrollo Sostenible (ODS), Agenda 2030, etc.

\section{Regulación y autorregulación de los medios}

La reforma mediática se da en muchas ocasiones desde el interior de los propios medios, a partir de políticas públicas que garantizan la libertad de expresión y sus límites (privacidad, discurso del odio, etc.), o mediante propuestas que invitan a transformar el periodismo existente: periodismo de investigación, público (public journalism), social, para la paz, ambiental, slow journalism, entre otros (A révalo, 2014). Por otra parte, desde la ética de la comunicación, se alienta a profundizar en mecanismos de autorregulación como organismos independientes (consejos de prensa y del audiovisual, ómbusdman, sindicatos y gremios, etc.) o trabajos sobre calidad y buenas prácticas periodísticas: manuales de estilo, códigos deontológicos, recomendaciones, etc. (Mauri-Ríos y Ramón-Vegas, 2015).

\section{Comunicación estratégica de organizaciones formales}

Esta es una de las áreas de trabajo clásicas de la disciplina y hace referencia a la tradición más institucionalista, profesionalizada y formal de la CDCS, o, en otras palabras, cómo diversas organizaciones -en especial ONG y agencias- estructuran su comunicación para favorecer cambios: bien mediante políticas y campañas para situar determinados temas en la agenda pública, bien con la participación ciudadana en proyectos con metodologías como la investigación participativa o el eduentretenimiento (edutainment). Desde esta perspectiva, se alienta a que la CDCS no deba ser entendida como una mera réplica de la comunicación corporativa u organizacional, dado que muchas organizaciones comunitarias llevan años desarrollando planteamientos diferenciados del ámbito corporativo (McAnany, 2010): publicidad social, marketing con causa, advocacy, sensibilización, promoción de la salud, etc. 


\section{Educomunicación}

Relacionada con lo anterior, la educación en medios ha acabado convirtiéndose, por derecho propio, en un campo específico debido a su extenso volumen de estudios. No obstante, sus raíces se relacionan con las de la CDCS, en particular en el ámbito latinoamericano e hispanohablante, donde teóricos de ambas subdisciplinas citan como inspiración común la obra de Paulo Freire (1970/2002) o las primeras prácticas de educomunicación popular en América Latina: radioescuelas, medios populares y comunitarios, etc. Con esta etiqueta se hace referencia a investigaciones que, desde un enfoque más pedagógico o comunicacional, inciden en uno de estos tres ejes: (1) mejorar los procesos pedagógicos mediante la incorporación de tecnologías mediáticas; (2) educar para la recepción crítica audiovisual y multimedia y (3), en su nivel más avanzado, contribuir a que las poblaciones se apropien de las herramientas informacionales a fin de crear productos autónomos, lo que se vincula con el objeto de la "apropiación" descrito más adelante. Aquí, el origen de la intervención puede ser más externo, vertical o implementado desde escuelas u organismos públicos, o más interno u horizontal, mediante prácticas de educación informal de sectores populares, movimientos sociales, asociaciones culturales y vecinales, etc.

\section{Vigilancia mediática}

Además de reformas al interior de los medios, también hay iniciativas orientadas a supervisar y denunciar el incumplimiento de la función de servicio público de los medios, por ejemplo, cuando estigmatizan a determinadas poblaciones, o cuando se incurre en discurso del odio o publicidad degradante con respecto a determinados colectivos. Aquí destacan organizaciones de distinta índole y con participación, en mayor o menor grado, de la ciudadanía: observatorios y veedurías de medios, sindicatos y gremios independientes, asociaciones de consumidores y telespectadores, herramientas virtuales de verificación del periodismo, etc.

\section{Recepción y resignificación}

En este punto, se engloban dos tradiciones de estudios que enfatizan en el poder de negociación y resignificación de las audiencias. Por un lado, los estudios de recepción de corte anglosajón (p. ej., Stuart Hall, Ang Lee) o latinoamericano (p. ej., Guillermo Orozco, Jesús Martín Barbero), que enfatizan en la capacidad negociadora o productora de significados alternativos por parte de las audiencias y, por otro, las múltiples prácticas de guerrilla comunicacional, reapropiación, subversión (subverting) y contrainformación a cargo de activistas, artivistas y movimientos sociales. Aunque este objeto no cuenta con una tradición demasiado extensa ni asociada a la CDCS, son cada vez más frecuentes los procesos en los que la ciudadanía se apropia de las representaciones mediáticas o culturales dominantes para ironizarlas o subvertirlas desde una perspectiva crítica y desveladora de sus significados: memes, contrapropaganda, brandalism, etc. (Lekakis, 2017).

\section{Apropiación}

Este es el nivel más amplio de participación ciudadana y ejercicio efectivo del derecho a la comunicación. Históricamente, la CDCS ha enfatizado en el papel de los medios comunitarios como tradición conformadora del enfoque participativo de la disciplina (Beltrán, 1993), y en la que cabe enmarcar desde medios con distintas fórmulas de organización y financiación (medios libres, comunitarios, cooperativos, educativos, etc.), o formatos como el papel (periódicos, fanzines, libelos, etc.) o el audiovisual (radio comunitaria, videoactivismo, vídeo participativo, etc.). A estos medios más tradicionales, hay que sumar un 
contingente reciente de estudios sobre TIC (Internet, blogs, redes sociales, telefonía móvil) y movimientos sociales, desde marcos renovados como la ética hacker, la tecnopolítica, la soberanía tecnología, los comunes o la neutralidad de la red. No obstante, la mayoría de estos últimos estudios no suele adscribirse a la tradición de la CDCS sino que, como antes se dijo, está tendiendo a conformar un campo propio de estudios, en especial en Europa y EE. UU. La tabla 2 resume los principales objetos que comprende el modelo diseñado, atendiendo al cruce entre las dimensiones analíticas descritas.

TABLA 2

Modelo para el análisis de la CDCS

\begin{tabular}{|c|c|c|c|}
\hline $\begin{array}{l}\text { Origen de la } \\
\text { intervención }\end{array}$ & $\begin{array}{l}\text { Grado de } \\
\text { participación de la } \\
\text { ciudadanía }\end{array}$ & Objeto de investigación & Descripción y subtemas que comprende \\
\hline \multirow[t]{3}{*}{$\begin{array}{l}\text { Desde el interior de } \\
\text { los medios }\end{array}$} & Bajo & $\begin{array}{l}\text { Acceso a medios y } \\
\text { tecnologías }\end{array}$ & $\begin{array}{l}\text { Investigaciones sobre acceso ciudadano y políticas de acceso a } \\
\text { medios y TIC y estudios sobre brecha informacional y digital }\end{array}$ \\
\hline & Bajo & $\begin{array}{l}\text { Representación de la } \\
\text { ciudadanía en los medios }\end{array}$ & $\begin{array}{l}\text { Representación de la ciudadanía y de los grupos vulnerables, de los } \\
\text { movimientos sociales o de cuestiones relacionadas con derechos } \\
\text { humanos, objetivos del desarrollo, etc. }\end{array}$ \\
\hline & Bajo & $\begin{array}{l}\text { Regulación y } \\
\text { autorregulación de los } \\
\text { medios }\end{array}$ & $\begin{array}{l}\text { Ética de la comunicación, códigos y organismos autorreguladores, } \\
\text { filosofias y prácticas para la mejora del ejercicio periodístico, } \\
\text { políticas y regulaciones para la participación, la diversidad cultural, } \\
\text { etc. }\end{array}$ \\
\hline \multirow[t]{3}{*}{$\begin{array}{l}\text { Desde el exterior de } \\
\text { los medios }\end{array}$} & Medio & $\begin{array}{l}\text { Comunicación estratégica de } \\
\text { organizaciones ciudadanas }\end{array}$ & $\begin{array}{l}\text { Estrategias comunicacionales de desarrollo de agencias de } \\
\text { cooperación y ONG, con mayor o menor grado de participación } \\
\text { ciudadana. }\end{array}$ \\
\hline & Medio & Educomunicación & $\begin{array}{l}\text { Procesos y prácticas de educación mediática, con mayor o menor } \\
\text { grado de apropiación tecnológica. }\end{array}$ \\
\hline & Medio & Vigilancia mediática & $\begin{array}{l}\text { Fiscalización externa del sistema de medios por parte de } \\
\text { asociaciones de espectadores, observatorios de medios, webs de } \\
\text { quejas, etc. }\end{array}$ \\
\hline \multirow[t]{2}{*}{$\begin{array}{l}\text { Desde el exterior de } \\
\text { los medios }\end{array}$} & Alto & Recepción y resignificación & $\begin{array}{l}\text { Prácticas de recepción de contenidos mediáticos y estrategias de } \\
\text { contrainformación, subversión y guerrilla informacional. }\end{array}$ \\
\hline & Alto & Apropiación & $\begin{array}{l}\text { Prácticas de apropiación ciudadana de medios alternativos } \\
\text { tradicionales (p. ej., radio comunitaria) y nuevos medios apoyados } \\
\text { en TIC. }\end{array}$ \\
\hline
\end{tabular}

Fuente: elaboración propia

Por último, cabe señalar tres consideraciones al modelo. En primer lugar, se ha descrito un continuum de prácticas de reforma distintas, a modo de "tipos-ideales" (Weber, 1982) o formas puras situadas entre dos extremos: por un lado, reformas mediáticas exógenas a la ciudadanía y desde el interior de los medios tradicionales ("acceso" y “representación”), y por otro, iniciativas endógenas y desde el exterior de los medios ("recepción y resignificación" y "apropiación"). Junto a estas se sitúan otras modalidades intermedias con mayor o menor grado de participación ciudadana ("comunicación estratégica de organizaciones formales", "vigilancia ciudadana" y "educomunicación"). En segundo lugar, algunos de estos objetos no siempre se han asociado a la tradición de estudios de la CDCS, pese a que intentan responder a la pregunta acerca de la relación entre comunicación, ciudadanía y cambio social. Se hace referencia, en particular, a los objetos de "acceso", “representación”, “educomunicación”, "rendición de cuentas", "vigilancia” y "recepción o reapropiación". En este sentido, no se pretende que la CDCS se convierta en un paraguas multiabarcante en el que se enmarquen nuevos objetos de la investigación comunicacional, sino que nuestro objeto último es el de clarificar diferentes temas y niveles que podrían ser abordados desde la óptica del campo. Por último, a fin de reducir la complejidad, el modelo se ha focalizado sobre todo en prácticas y procesos mediáticos, por lo que se excluyen expresiones culturales y artísticas de tipo diverso que también están relacionadas con el área: teatro del oprimido, canción protesta, performance, música, cartelismo, etc. 


\section{Conclusiones}

Este estudio ha intentado tender puentes entre el campo de la CDCS y el concepto de reforma mediática, que ayuda a ensanchar el ámbito, incorporando debates de la economía política de la comunicación y trabajos sobre movimientos sociales. A partir de este diálogo, se ha pretendido sistematizar cuáles los diferentes objetos que podría abarcar la relación entre comunicación, ciudadanía y cambio social. En este sentido, se enfrentó un panorama temático amplio y disímil, en el que se incluyen objetos macro o micro, más institucionalistas o basales y de carácter más vertical u horizontal. Además, se diseñó un modelo construido en torno a dos variables: origen de la intervención (desde el interior o el exterior de los medios) y grado de participación de la ciudadanía (alta, media o baja).

Frente a la fragmentación creciente del conocimiento comunicacional, este modelo intenta favorecer la interdisciplinariedad y el diálogo entre áreas de la comunicación que no siempre deberían pensarse como entes aislados, como es el caso de la educomunicación y CDCS. Por otra parte, el modelo se orienta a plantear diálogos interregionales mediante la lógica explicativa de tipos-ideales weberianos. Así, planteamos observar la CDCS desde la pregunta de qué puede hacer la ciudadanía organizada para transformar sus medios, desestimando cualquier tipo de enfoque mediacéntrico e instrumentalista y adscribiéndonos a marcos como el de las mediaciones (Martín Barbero, 1987) y las prácticas mediáticas (Couldry, 2012), que invitan a descartar perspectivas instrumentales y tecnoutópicas y a poner el foco en la agencia de los sujetos y los contextos particulares de cada práctica.

Por último, se considera que el modelo puede contribuir al diálogo entre los estudios del Norte y el Sur del planeta hacia la construcción de estudios mediáticos comparados. Esta misión ha sido acometida por importantes volúmenes publicados en tiempos recientes tanto en CDCS (Gumucio y Tufte, 2006) como en comunicación alternativa (Atton, 2015; Downing, 2010), si bien no desde la perspectiva estricta de los estudios comparados, que invita a desarrollar un enfoque transcultural para desvelar culturas mediáticas regionales o culturales más allá de las fronteras habituales del Estado-nación (Hepp y Couldry, 2009, p. 32). A nuestro parecer, el reto pasaría ahora por testar dicho modelo, por ejemplo, en el ámbito del diseño de currículos educativos o de cara a acometer estudios metateóricos en el área, mediante análisis de contenidos o bibliométricos.

\section{Referencias}

Alfaro, R. M. (2006). Otra brújula: innovaciones en comunicación y desarrollo. Lima: Editorial de la Asociación de Comunicadores Sociales Calandria.

Álvarez, M. (2017). Demandas de reforma mediática y momento populista. La circulación de las propuestas de democratización de los medios en el espacio político post-bipartidista. IC. Revista de Información y Comunicación, 14, 121-157. https://doi.org/10.12795/IC.2017.i01.05

Ángel, A. (2013). Los "campos" de la comunicación organizacional: perspectivas latinoamericanas y norteamericanas. Filo de Palabra, 15, 9-26. Recuperado de http://revistasum.umanizales.edu.co/ojs/index.php/filodepalabra/ar ticle/view/1001/1115

Arévalo, A. (2014). Periodismo y comunicación para la paz. Indicadores y marco regulatorio. Commons. Revista de Comunicación y Ciudadanía Digital, 3(1), 57-92. Recuperado de https://revistas.uca.es/index.php/cayp/articl e/view/3075

Atton, C. (ed.). (2015). The Routledge companion to alternative and community media. Nueva York: Routledge.

Barassi, V. (2016). Contested visions: Digital discourses as empty signifiers from the 'network' to 'big data'. Communication and the Public, 1(4), 423-435. https://doi.org/10.1177/2057047316680220 
Barranquero, A., y Ángel, A. (2015). La producción académica sobre comunicación, desarrollo y cambio social en las revistas científicas de América Latina. Signo y Pensamiento, 34(67), 30-58. https://doi.org/10.11144/Javeriana .syp34-67.pacd

Barranquero, A., y Sáez-Baeza, C. (2015). Comunicación y buen vivir. La crítica descolonial y ecológica a la comunicación para el desarrollo y el cambio social. Palabra Clave, 18(1), 41-82. https://doi.org/10.5294/pacl a.2015.18.1.3

Beltrán, L. R. (febrero, 1993). Comunicación para el desarrollo en Latinoamérica: una evaluación sucinta al cabo de cuarenta años. Discurso de apertura en la inauguración de la IV Mesa Redonda sobre Comunicación y Desarrollo, Instituto para América Latina, Lima. Recuperado de http://www.bantaba.ehu.es/sociedad/files/view/comunic acion_para_el_desarrollo_en_latinoamerica.pdf?revision\%5Fid=62744\&package\%5Fid=33044

Bourdieu, P. (2003). Campo de poder, campo intelectual: itinerario de un concepto. Buenos Aires: Editorial Quadrata.

Calabrese, A. (2004). The promise of civil society: A global movement for communication rights. Continuum: Journal of Media \& Cultural Studies, 18(3), 317-329. https://doi.org/10.1080/1030431042000256081

Cammaerts, B. (2017). Communication freedoms versus communication rights: Discursive and normative struggles within civil society and beyond. En H. Tumber y S. Waisbord (eds.), The Routledge companion to media and human rights (pp. 50-59). Abingdon, MD: Routledge.

Carpentier, N. (2011). Media and participation. A site of ideological-democratic struggle. Bristol, UK: Intellect.

Chaparro, M. (2015). Claves para repensar los medios y el mundo que habitamos. La distopía del desarrollo. Bogotá: Ediciones desde Abajo.

Couldry, N. (2010). Why voice matters. Culture and politics after neoliberalism: Thousand Oaks, CA: Sage.

Couldry, N. (2012). Media, society, world: Social theory and digital media practice. Cambridge, MA: Polity Press.

Crowther, C., Thibault, S., Salamon, E., y King, G. (2016). Introduction: Whose crisis? Journalism is not just for journalists and policy is not just for wonks. En M. Gasher, C. Brin, C. Crowther, G. King, E. Salamon y S. Thibault (Dirs.), Journalism in crisis: Bridging theory and practice for democratic media strategies in Canada (pp. 3-23). Toronto: University of Toronto Press.

Downing, J. D. (ed.). (2010). Encyclopedia of social movement media. Thousand Oaks, CA: Sage.

Dreher, T. (2010). Speaking up or being heard? Community media interventions and the politics of listening. Media, Culture \& Society, 32(1), 85-103. https://doi.org/10.1177/0163443709350099

El-Issawi, F. (2016). Arab national media and political change: Recording the transition. Londres: Palgrave Macmillan.

Enghel, F. (2015). Towards a political economy of communication in development? [Número especial]. Nordicom Review, 36, 11-24. Recuperado de https://www.nordicom.gu.se/sites/default/files/kapitel-pdf/nordicom_revi ew_36_2015_special_issue_pp._11-24.pdf

Fenton, N., y Freedman, D. (2014). The politics and possibilities of media reform. Lessons from the UK. En T. Miller (ed.), The Routledge companion to global popular culture (pp. 458-470). Nueva York/Londres: Routledge.

Ferron, B. (2012). Les médias alternatifs: "contre-culture" ou "sous-culture"? Les luttes de dé-légitimation de la communication contestataire à travers l'étude de publications académiques et militantes (Tesis doctoral no publicada). Université de Rennes 1, Bretaña.

Fones-Wolf, E. A. (2006). Waves of opposition: Labor, business, and the struggle for democratic radio. Urbana, IL: University of Illinois.

Franklin, M. I. (2016). Mobilizing for net rights: The IRPC Charter of Human Rights and Principles for the Internet. En D. Freedman, J. A. Obar, C. Martens y R. W. McChesney (eds.), Strategies for media reform: International perspectives (pp. 72-91). Nueva York: Fordham University.

Fraser, N. (2017). ¿De la redistribución al reconocimiento? Dilemas de la justicia en la era "postsocialista". En J. Butler y N. Faser (eds.), ¿Reconocimiento o redistribución? Un debate entre el marxismo y el feminismo (pp. 23-66). Madrid: Traficantes.

Freedman, D. (2014). The contradictions of media power. Nueva York: Bloomsbury. 
Freedman, D., Obar, J. A., Martens C., y McChesney, R. W. (eds.). (2016). Strategies for media reform: International perspectives. Nueva York: Fordham University.

Freedman, D., y Obar, J. A. (2016). Media reform. An overview. En D. Freedman, J. A. Obar, C. Martens y R. W. McChesney (eds.), Strategies for media reform: International perspectives (pp. 3-18). Nueva York: Fordham University.

Freire, P. (2002). Pedagogía del oprimido (16.a ed.). Madrid: Siglo XXI.

García García, J. (2017). Transformaciones y aprendizajes de las radios comunitarias en España: hacia un modelo de radio inclusiva. Disertaciones, 10(1), 30-41. https://doi.org/10.12804/revistas.urosario.edu.co/disertaciones/a. 4535

Gee, J. P. (2010). New digital media and learning as an emerging area and "worked examples" as one way forward. Cambridge, MA: Massachusetts Institute of Technology.

Gumucio, A., y Tufte, T. (eds.). (2006). Communication for social change anthology: Historical and contemporary readings. South Orange, NJ: Communication for Social Change Consortium.

Hackett, A. (2000). Taking back the media: Notes on the potential for a communicative democracy movement. Studies in Political Economy, 63, 61-86. https://doi.org/10.1080/19187033.2000.11675233

Hackett, R. (2014). Media democratization in Canada: A movement comes into its own. New Left Project. Recuperado de http://www.newleftproject.org/index.php/site/article_comments/media_democratisation_in _canada_a_movement_comes_into_its_own

Hackett, R., y Carroll, W. (2006). Remarking media. The struggle to democratize public communication. Abingdon, IL: Routledge.

Hamelink, C. (2014). Communication rights and the history of ideas. En C. Padovani y A. Calabrese (eds.), Communication rights and social justice. Historical accounts of transnational mobilizations (pp. 17-28). Nueva York: Palgrave/International Association for Media and Communication Research.

Hepp, A., y Couldry, N. (2009). What should comparative media research be comparing? Towards a transcultural approach to 'media cultures'. En D. K. Thussu (ed.), Internationalizing media studies (pp. 32-47). Londres: Routledge.

Jacobson, T. L. (2016). Amartya sen's capabilities approach and communication for development and social change. Journal of Communication, 66(5), 789-810. https://doi.org/10.1111/jcom.12252

Krishnan, A. (2009). What are academic disciplines? Some observations on the disciplinarity vs. interdisciplinarity debate (National Centre for Research Methods Working Paper Series 03/09). Recuperado de http://eprints.ncrm.ac.u $\mathrm{k} / 783 / 1 /$ what_are_academic_disciplines.pdf

Lakatos, I. (1978). La metodología de los programas de investigación. Madrid: Alianza.

Lekakis, E. J. (2017). Culture jamming and brandalism for the environment: The logic of appropriation. Popular Communication, 15(4), 311-327. https://doi.org/10.1080/15405702.2017.1313978

Lennie, J., y Tacchi, J. (2013). Evaluating communication for development. A framework for social change. Londres: Routledge.

Lie, R., y Servaes, J. (2015). Disciplines in the field of communication for development and social change. Communication Theory, 25, 244-258. https://doi.org/10.1111/comt.12065

Manyozo, L. (2012). Media, communication and development: Three approaches. Thousand Oaks, CA: Sage.

Marí Sáez, V. (2011). Comunicar para transformar, transformar para comunicar. Tecnologías de la información desde una perspectiva de cambio social. Madrid: Editorial Popular.

Martín Barbero, J. (1987). De los medios a las mediaciones. Comunicación, cultura y hegemonía. México: Gili.

Martínez, M., y Sierra, F. (2012). Comunicación y desarrollo. Prácticas comunicativas y empoderamiento local. Barcelona: Gedisa.

Mauri-Ríos, M., y Ramón-Vegas, X. (2015). Nuevos sistemas de rendición de cuentas de la información periodística. Exploración del escenario online español. El Profesional de la Información, 24(4), 380-389. https://doi.org/10 $.3145 /$ epi.2015.jul.04 
Media Reform. (s. f.). Recuperado de https://www.mediareform.org.uk

McAnany, E. (2010). Communication for development and social change: New millennium. A Quarterly Review of Communication, 29(3), 1-43. Recuperado de http://cscc.scu.edu/trends/v29/CRT_v29_n3_September2010. pdf

McChesney, R. W. (1999). Rich media, poor democracy: Communication politics in dubious times. Urbana, IL: University of Illinois Press.

McChesney, R. W. (2016). Preface. En D. Freedman, J. A. Obar, C. Martens y R. W. McChesney (eds.), Strategies for media reform: International perspectives (pp. ix-xvi). Nueva York: Fordham University Press.

Melkote, S. R., y Steeves, H. L. (2001). Communication for development in the Third World: Theory and practice for empowerment. Thousand Oaks, CA: Sage.

Montuori, A. (2005). Literature review as creative inquiry: Reframing scholarship as a creative process. Journal of Transformative Education, 3(4), 374-393. https://doi.org/10.1177/1541344605279381

Napoli, P. (2007). Public interest media activism and advocacy as a social movement: A review of the literature (McGannon Center Working Paper Series no. 21). Recuperado de http://fordham.bepress.com/mcgannon_w orking_papers/21

Obregón, R., y Tufte, T. (2017). Communication, social movements and collective action. Research agenda in communication for development and social change. Journal of Communication, 67, 635-645. https://doi.org/1 $0.1111 /$ jcom. 12332

Ogan, C., Bashir, M., Camaj, L., Luo, Y., Gaddie, B., Pennington, R., ... Salih, M. (2009). Development communication. The state of research in an era of ICTs and globalization. International Communication Gazette, 71(8), 655-670. https://doi.org/10.1177/1748048509345060

Open Media. (s. f.). Recuperado de https://openmedia.org

Padovani, C., y Calabrese, A. (eds.). (2014). Communication rights and social justice: Historical accounts of transnational mobilizations. Londres: Palgrave Macmillan.

Pickard, V. (2015). America's battle for media democracy. The triumph of corporate libertarianism and the future of media reform. Nueva York: Cambridge University Press.

Pickard, V., y Yang, G. (eds.). (2017). Media activism in the digital age: Charting an evolving field of research. Londres: Routledge.

Prieto, C. (2013). El Foro Andaluz de Comunicación como caso de empoderamiento ciudadano ante la futura ley audiovisual de ámbito económico. Communication Papers, 3, 22-37. Recuperado de https://www.academia.edu/5911176/EL_FORO_ANDALUZ_DE_COMUNICACI\%C3\%93N_COM O_CASO_DE_EMPODERAMIENTO_CIUDADANO_ANTE_LA_FUTURA_LEY_AUDIOVISUAL _DE_\%C3\%81MBITO_AUTON\%C3\%93MICO

Quarry, W., y Ramírez, R. (2009). Communication for another development: Listening before telling. Londres: Zed Books.

Rodríguez, C., Ferron, B., y Shamas, K. (2014). Four challenges in the field of alternative, radical and citizens' media research. Media, Culture \& Society, 36(2), 1-17. https://doi.org/10.1177/0163443714523877

Rodríguez, C., Obregón, R., y Vega, J. (2002). Estrategias de comunicación para el cambio social. Quito: Friederich Ebert Stiftung.

Rogers, E. M. (1962). Diffusion of innovations. Glencoe, IL: Free Press.

Sarikakis, K., y Rodríguez-Amat, J. -R. (2014). Intellectual property law change and process: The case of Spanish Ley Sinde as policy laundering. First Monday, 19(3). https://doi.org/10.5210/fm.v19i3.4854

Segura, M. S. (2011). La reforma comunicacional: la construcción de sus argumentos y condiciones de posibilidad a partir de 2001. Estudios, 26, 125-142. https://www.researchgate.net/profile/Maria_Segura25/publication/277225032_La-reforma-comun icacional-La-construccion-de-sus-argumentos-y-condiciones-de-posibilidad-a-partir-de-2001.pdf 
Segura, M. S., y Waisbord, S. (2016). Media movements. Civil society and media policy reform in Latin America. Londres: Zed Books.

Servaes, J. (ed.). (2019). Handbook of communication for development and social change. Singapore: Springer.

Servaes, J., Polk, E., Shi, S., Reilly, D., y Yakupitijage, T. (2012). Towards a framework of sustainability indicators for 'communication for development and social change' projects. International Communication Gazette, 74(2), 99-123. https://doi.org/10.1080/09614524.2012.634177

Sieberg, F. S., Peterson, T., y Schramm, W. (1956). Four theories of the press. Urbana, IL: University of Illinois Press.

Stephansen, H. (2017). Media activism as movement? Collective identity formation in the World Forum of Free Media. Media and Communication, 5(3), 59-66. https://doi.org/10.17645/mac.v5i3.1034

The Media Democracy Project. (s. f.). Recuperado de http://mediademocracyproject.ca

Tichenor, P. J., Donohue, G. A., y Olien, C. N. (1970). Mass media flow and differential growth in knowledge. Public Opinion Quarterly, 34, 159-170. https://doi.org/10.1086/267786

Tucho, F. (2006). La sociedad civil ante el sistema mediático. El movimiento de reforma de los medios en Estados Unidos. Zer. Revista de Estudios de Comunicación, 11(20), 299-317. Recuperado de https://www.ehu.eus/ojs/ index.php/Zer/article/view/3768/3398

Tufte, T. (2017). Communication and social change: A citizen perspective. Cambridge, MA: Polity Press.

Weber, M. (1982). Ensayos sobre metodología sociológica. Buenos Aires: Amorrortu.

Wilkins, K. G., Tufte, T., y Obregón, R. (eds.). (2014). The handbook of development communication and social change. Oxford: Wiley.

Zallo, R. (2016). Tendencias en comunicación: cultura digital y poder. Barcelona: Gedisa.

\section{Notas}

1 En el ámbito internacional, también ha habido en tiempos recientes una eclosión de monográficos. En Estados Unidos, destacan el Journal of Communication (vol. 67, n. ${ }^{\circ}$, "Communication and collective action", 2018) y Communication Theory (vol. 25, n. ${ }^{\circ}$ 2, "Advocacy and communication for social change", 2015). En Latinoamérica, Cuadernos.info (n. ${ }^{\circ}$ 36, "Comunicación y desigualdad", 2015), Diálogos de la Comunicación (Edición 92, "Balance y presente de los estudios en comunicación, ciudadanía y cambio social, 2016), Disertaciones (vol. 10, n. ${ }^{\circ}$, "Comunicación y tercer sector”, 2017) o Razón y Palabra (vol. 18, n. ${ }^{\circ}$ 1, "Comunicación y ciudadanía”, 2014 y vol. 20, n. ${ }^{\circ}$ 4, "Dinámica de la cultura, de la ciudadanía y de la inclusión social”, 2016). Ya en España, en el intervalo de dos años, se publicaron monográficos en Comunicar (n. ${ }^{4}$ 7, titulado "Comunicación, sociedad civil y cambio social”, 2016); Cultura, Lenguaje y Representación (vol. 15, "Comunicación y cambio social”, 2016) o Icono 14 (vol. 16, n. ${ }^{\circ}$ 1, “Comunicación, poder y cambio social”, 2018).

2 Un buen acercamiento a las diferencias entre la libertad de expresión y el nuevo derecho a la comunicación quedan explicitadas en trabajos como el de Cammaerts (2017) o Hamelink (2014).

3 Tichenor, Donohue y Olien (1970) inauguraron una extensa tradición de trabajos en torno al diferencial de conocimiento, que ayudó a complejizar la CDCS al poner el foco, ya no en el papel de los medios para favorecer el desarrollo, sino en la capacidad que tienen los individuos para acceder e interpretar las informaciones de los medios. Esta hipótesis plantea que los medios tienden a reproducir y acentuar las desigualdades culturales entre los estratos más altos y más bajos de la población, a diferencia de lo que venían señalando las teorías de la modernización, que proponían una ecuación simplista entre el aumento del número de medios en una región y su desarrollo económico y político.

* Artículo de investigación científica.Este trabajo se enmarca en las líneas habituales de investigación de la Red de Investigación en Comunicación Comunitaria, Alternativa y Participativa (RICCAP) (http://www.riccap.org) y en el proyecto I+D financiado "Mapas de la investigación en comunicación en las universidades españolas de 2007 a 2018” (ref. PGC2018-093358-B-I00).

\section{Licencia Creative Commons CC BY 4.0}

Cómo citar este artículo: Barranquero Carretero, A. (2019). Comunicación, ciudadanía y cambio social. Diseño de un modelo de investigación y acción para democratizar la comunicación desde la noción de reforma mediática. Signo y Pensamiento, 38(75). https://doi.org/10.11144/Javeriana.syp38-75.cccs 